SELECCIONES MATEMÁTICAS
Universidad Nacional de Trujillo
ISSN: $2411-1783$ (Online)
Vol. 03(02): $107-112(2016)$

\title{
Convexidad Estricta a Través de normas equivalentes en espacios de Banach Separables.
}

\author{
Strict Convexity Through Equivalent Norms in Separables Banach Spaces. \\ Willy Zubiaga Vera*
}

Received, Jun. 18, 2016

Accepted, Sept. 22, 2016.

DOI: http: //dx.doi.org/10.17268/sel.mat.2016.02.06

\begin{abstract}
Resumen
Sea E un espacio de Banach separable con norma $\|$.$\| . En el presente trabajo, se tiene como objetivo$ construir una norma $\|.\|_{1}$ que sea equivalente a $\|$.$\| en E$, tal que $\|.\|_{1}$ estrictamente convexa. Además se demuestra que su norma dual conjugada es también estrictamente convexa.

Palabras clave. Convexidad estricta, norma dual, espacios de Banach separable.
\end{abstract}

\begin{abstract}
Let $E$ be a separable Banach space with norm $\|$.$\| . In the present work, the objective is to construct a$ norm $\|.\|_{1}$ that is equivalent to $\|$.$\| in E$, such that $\|.\|_{1}$ is strictly convex. In addition it is shown that its dual conjugate norm is also strictly convex..

Keywords. Strict convexity, dual norm, separable Banach spaces.
\end{abstract}

1. Introducción. En esta sección se introduce las herramientas esenciales para demostrar nuestro resultado.

Sea $E$ un espacio normado dotado de la norma $\|$.$\| . Recordemos que la topología débil en el espacio$ normado $E$ es denotada por $\sigma\left(E, E^{*}\right)$, y es la topología generada por los funcionales continuos $f \in E^{*}$. Además, para cada $x_{0} \in E$, los conjuntos de la forma

$$
V_{J, \epsilon}=\left\{x \in E:\left|\left\langle f_{i}, x\right\rangle-\left\langle f_{i}, x_{0}\right\rangle\right|<\epsilon \text { para todo } i \in J\right\}
$$

donde $J$ es un conjunto finito, $f_{i} \in E^{*}$ para todo $i \in J$ y $\epsilon>0$, forman una base de vecindades abiertas de $x_{0}$ para la topología débil.

Sea $E^{*}$ el dual de $E$. La topología débil-* en $E^{*}$, es denotada por $\sigma\left(E^{*}, E\right)$, es la topología en $E^{*}$ generada por las funciones que pertenecen al conjunto $J_{E}=\left\{J_{E}(x): x \in E\right\}$, esto es, por las funciones

$$
f \in E^{*} \rightarrow J_{E}(x)(f)=\langle f, x\rangle
$$

donde $x \in E$. Para cada $f_{0} \in E^{*}$, los conjuntos de la forma

$$
W_{J, \epsilon}=\left\{f \in E^{*}:\left|\left\langle f, x_{i}\right\rangle-\left\langle f_{0}, x_{i}\right\rangle\right|<\epsilon \text { para todo } i \in J\right\}
$$

donde $J$ es un conjunto finito, $x_{i} \in E$ para todo $i \in J$ y $\epsilon>0$, forman una base de vecindades abiertas de $f_{0}$ en la topología débil-* (ver [1]).

* Departamento de Matemáticas-Universidad Nacional de Trujillo (wizuve@hotmail.com. 
Recordemos que, dado un espacio normado $E, B_{E}$ representa la bola unitaria cerrada de $E$, es decir

$$
B_{E}=\{x \in E:\|x\| \leq 1\}
$$

Teorema 1. (Teorema de Banach-Alaoglu-Bourbaki [1]) Para todo espacio normado E, la bola $B_{E^{*}}$ es compacta en la topología débil-* $\sigma\left(E^{*}, E\right)$ de $E^{*}$

Se dice que $E$ es separable, si existe un subconjunto denso $D \subset E$ que es denso y numerable en $E$.

Teorema 2. [1] Sea E un espacio de Banach. Entonces

1. Si $E^{*}$ es separable, entonces E es separable.

2. E es reflexivo y separable sí y sólo sí $E^{*}$ es reflexivo y separable.

3. Si E es separable, entonces $B_{E^{*}}$ es metrizable en la topología débil-* $\sigma\left(E^{*}, E\right)$.

4. Si $E^{*}$ es separable, entonces $B_{E}$ es metrizable en la topología débil $\sigma\left(E, E^{*}\right)$.

Un espacio de Banach es uniformemente convexo si $\forall \epsilon>0, \exists \delta>0$ tal que

$$
x, y \in E, \quad\|x\| \leq 1,\|y\| \leq 1 \text { y }\|x-y\| \geq \epsilon \Longrightarrow\left\|\frac{x+y}{2}\right\| \leq 1-\delta .
$$

Teorema 3. (Milman-Pettis [1]) Todo espacio de Banach uniformemente convexo es reflexivo.

Nota 1.1. Todo espacio de Hilbert $H$ es uniformemente convexo. En efecto, dados $x, y \in B_{H}$ y $0<$ $\epsilon \leq 2$, por la Ley del paralelogramo, se tiene que si $\|x-y\| \geq \epsilon$, entonces

$$
\left\|\frac{x+y}{2}\right\|^{2}=\frac{\|x\|^{2}}{2}+\frac{\|y\|^{2}}{2}-\frac{\|x-y\|^{2}}{4} \leq 1-\frac{\epsilon^{2}}{4} .
$$

Basta tomar $\delta=1-\left(1-\epsilon^{2} / 4\right)^{1 / 2}>0$

2. Resultado. Sea $\left(a_{n}\right) \subset B_{E}$ un subconjunto denso de $B_{E}$ con respecto a la topología fuerte. Sea $\left(b_{n}\right) \subset B_{E^{*}}$ un subconjunto numerable de $B_{E^{*}}$ que es denso en $B_{E^{*}}$ para la topología débil-* $\sigma\left(E^{*}, E\right)$. Se debe notar que, puesto que $E$ es un espacio de Banach separable, entonces $B_{E^{*}}$ es un espacio métrico compacto para la topología $\sigma\left(E^{*}, E\right)$, por lo tanto existe $\left(b_{n}\right) \subset B_{E^{*}}$ denso numerable (consecuencia del Teorema 1 y 2 ).

Considere el siguiente funcional

$$
\begin{aligned}
{[.]: E^{*} } & \rightarrow \mathbb{R} \\
f & \rightarrow[f]=\left(\sum_{n=1}^{\infty} \frac{1}{2^{n}}\left|\left\langle f, a_{n}\right\rangle\right|^{2}\right)^{1 / 2} .
\end{aligned}
$$

Notemos que

$$
\sum_{n=1}^{\infty} \frac{1}{2^{n}}\left|\left\langle f, a_{n}\right\rangle\right|^{2} \leq \sum_{n=1}^{\infty} \frac{1}{2^{n}}\|f\|^{2}\left\|a_{n}\right\|^{2} \leq \sum_{n=1}^{\infty} \frac{1}{2^{n}}<1,
$$

lo cual implica que $[f]$ esta bien definida.

Afirmación 1: [.] es una norma en $E^{*}$.

En efecto. Se debe notar que $[f] \geq 0 \forall f \in B_{E^{*}}$, además si $[f]=0$, entonces

$$
\left\langle f, a_{n}\right\rangle=0 \quad \forall n \in \mathbb{N} .
$$

Puesto que $\left\{a_{n}\right\}$ es denso en $E$ y $f$ es continua entonces $\langle f, a\rangle=0$ para cualquier $a \in E$, esto implica que $f=0$.

Sea $\lambda \in \mathbb{R}$ y $f \in B_{E^{*}}$ entonces

$$
\begin{aligned}
{[\lambda f] } & =\left(\sum_{n=1}^{\infty} \frac{1}{2^{n}}\left|\left\langle\lambda f, a_{n}\right\rangle\right|^{2}\right)^{1 / 2} \\
& =\left(|\lambda|^{2} \sum_{n=1}^{\infty} \frac{1}{2^{n}}\left|\left\langle f, a_{n}\right\rangle\right|^{2}\right)^{1 / 2} \\
& =|\lambda|\left(\sum_{n=1}^{\infty} \frac{1}{2^{n}}\left|\left\langle\lambda f, a_{n}\right\rangle\right|^{2}\right)^{1 / 2}=|\lambda|[f]
\end{aligned}
$$


Ahora se quiere probar que $[f+g] \leq[f]+[g]$ para todo $f, g \in B_{E^{*}}$. En efecto, sean

$$
a=\frac{\left|\left\langle f, a_{n}\right\rangle\right|}{[f]}, \quad b=\frac{\left|\left\langle g, a_{n}\right\rangle\right|}{[g]} .
$$

Por la desigualdad de Young se tiene

$$
\frac{\left|\left\langle f, a_{n}\right\rangle\right|}{[f]} \frac{\left|\left\langle g, a_{n}\right\rangle\right|}{[g]} \leq \frac{1}{2}\left(\frac{\left|\left\langle f, a_{n}\right\rangle\right|^{2}}{[f]^{2}}+\frac{\left|\left\langle g, a_{n}\right\rangle\right|^{2}}{[g]^{2}}\right)
$$

luego

$$
\begin{aligned}
\sum_{n=1}^{\infty} \frac{1}{2^{n}} \frac{\left|\left\langle f, a_{n}\right\rangle\right|}{[f]} \frac{\left|\left\langle g, a_{n}\right\rangle\right|}{[g]} & \leq \frac{1}{2}\left(\frac{1}{[f]^{2}} \sum_{n=1}^{\infty} \frac{1}{2^{n}}\left|\left\langle f, a_{n}\right\rangle\right|^{2}+\frac{1}{[g]^{2}} \sum_{n=1}^{\infty} \frac{1}{2^{n}}\left|\left\langle g, a_{n}\right\rangle\right|^{2}\right) \\
& \leq 1 .
\end{aligned}
$$

Por lo tanto

$$
\sum_{n=1}^{\infty} \frac{1}{2^{n}}\left|\left\langle f, a_{n}\right\rangle\right|\left|\left\langle g, a_{n}\right\rangle\right| \leq[f][g]
$$

Ahora, puesto que

$$
\begin{aligned}
\left|\left\langle f+g, a_{n}\right\rangle\right|^{2} & =\left|\left\langle f+g, a_{n}\right\rangle\right|\left|\left\langle f+g, a_{n}\right\rangle\right| \\
& \leq\left|\left\langle f, a_{n}\right\rangle\right|\left|\left\langle f+g, a_{n}\right\rangle\right|+\left|\left\langle g, a_{n}\right\rangle\right|\left|\left\langle f+g, a_{n}\right\rangle\right|
\end{aligned}
$$

entonces, se obtiene

$$
\sum_{n=1}^{\infty} \frac{1}{2^{n}}\left|\left\langle f+g, a_{n}\right\rangle\right|^{2} \leq \sum_{n=1}^{\infty} \frac{1}{2^{n}}\left|\left\langle f, a_{n}\right\rangle\right|\left|\left\langle f+g, a_{n}\right\rangle\right|+\sum_{n=1}^{\infty} \frac{1}{2^{n}}\left|\left\langle g, a_{n}\right\rangle\right|\left|\left\langle f+g, a_{n}\right\rangle\right|
$$

Combinando esto último con la ecuación (2.2) se tiene

$$
\begin{aligned}
\sum_{n=1}^{\infty} \frac{1}{2^{n}}\left|\left\langle f+g, a_{n}\right\rangle\right|^{2} \leq & \sum_{n=1}^{\infty} \frac{1}{2^{n}}\left|\left\langle f, a_{n}\right\rangle\right|\left|\left\langle f+g, a_{n}\right\rangle\right|+\sum_{n=1}^{\infty} \frac{1}{2^{n}}\left|\left\langle g, a_{n}\right\rangle\right|\left|\left\langle f+g, a_{n}\right\rangle\right| \\
\leq & \left(\sum_{n=1}^{\infty} \frac{1}{2^{n}}\left|\left\langle f, a_{n}\right\rangle\right|^{2}\right)^{1 / 2}\left(\sum_{n=1}^{\infty} \frac{1}{2^{n}}\left|\left\langle f+g, a_{n}\right\rangle\right|^{2}\right)^{1 / 2} \\
& +\left(\sum_{n=1}^{\infty} \frac{1}{2^{n}}\left|\left\langle g, a_{n}\right\rangle\right|^{2}\right)^{1 / 2}\left(\sum_{n=1}^{\infty}\left|\left\langle f+g, a_{n}\right\rangle\right|^{2}\right)^{1 / 2} \\
\left(\sum_{n=1}^{\infty} \frac{1}{2^{n}}\left|\left\langle f+g, a_{n}\right\rangle\right|\right)^{1 / 2} \leq & \left(\sum_{n=1}^{\infty} \frac{1}{2^{n}}\left|\left\langle f, a_{n}\right\rangle\right|^{2}\right)^{1 / 2}+\left(\sum_{n=1}^{\infty} \frac{1}{2^{n}}\left|\left\langle g, a_{n}\right\rangle\right|^{2}\right)^{1 / 2},
\end{aligned}
$$

asi $[f+g] \leq[f]+[g]$. Por lo tanto [.] es una norma.

Ahora, sea $f \in E^{*}$ y

$$
\|f\|_{1}=\left(\|f\|^{2}+[f]^{2}\right)^{1 / 2}
$$

donde $\|$.$\| denota la norma en el espacio E^{*}$

Afirmación 2: $\|\cdot\|_{1}$ es una norma equivalente a $\|\cdot\|$. Por lo anterior se tiene que $\|\cdot\|_{1}$ es norma. Ademas, como $a_{n} \in B_{E}$, entonces $\left\|a_{n}\right\| \leq 1$ para todo $n \in \mathbb{N}$, luego

$$
\begin{aligned}
\|f\| & \leq\|f\|_{1}=\left(\|f\|^{2}+\sum_{n=1}^{\infty} \frac{1}{2^{n}}\left|\left\langle f, a_{n}\right\rangle\right|^{2}\right)^{1 / 2} \\
& \leq\left(\|f\|^{2}+\sum_{n=1}^{\infty} \frac{1}{2^{n}}\|f\|^{2}\left\|a_{n}\right\|^{2}\right)^{1 / 2} \\
& \leq\left(\|f\|^{2}+\|f\|^{2} \sum_{n=1}^{\infty} \frac{1}{2^{n}}\right)^{1 / 2} \leq \sqrt{2}\|f\| .
\end{aligned}
$$


así se concluye la afirmación.

Afirmación 3: $\|.\|_{1}$ es estrictamente convexa. En efecto, se debe notar que [.] es inducida por un producto. En efecto, verifiquemos que [.] cumple la ley del paralelogramo se tiene

$$
\begin{aligned}
\sum_{n=1}^{\infty} \frac{1}{2^{n}}\left|\left\langle f+g, a_{n}\right\rangle\right|^{2} & +\sum_{n=1}^{\infty} \frac{1}{2^{n}}\left|\left\langle f-g, a_{n}\right\rangle\right|^{2} \\
& =\sum_{n=1}^{\infty} \frac{1}{2^{n}}\left(\left|\left\langle f, a_{n}\right\rangle\right|^{2}+2\left\langle f, a_{n}\right\rangle\left\langle g, a_{n}\right\rangle+\left|\left\langle g, a_{n}\right\rangle\right|^{2}\right) \\
& +\sum_{n=1}^{\infty} \frac{1}{2^{n}}\left(\left|\left\langle f, a_{n}\right\rangle\right|^{2}-2\left\langle f, a_{n}\right\rangle\left\langle g, a_{n}\right\rangle+\left|\left\langle g, a_{n}\right\rangle\right|^{2}\right) \\
& =2[f]^{2}+2[g]^{2} .
\end{aligned}
$$

Por lo tanto [.] es estrictamente convexa (ya que es inducida por un producto interno). Además, para todo $t \in[0,1]$ y para todo $f, g \in E^{*}$ se tiene

$$
\begin{aligned}
{[t f+(1-t) g]^{2}+} & t(1-t)[f-g]^{2} \\
= & \sum_{n=1}^{\infty} \frac{1}{2^{n}}\left|\left\langle t f+(1-t) g, a_{n}\right\rangle\right|^{2}+t(1-t) \sum_{n=1}^{\infty} \frac{1}{2^{n}}\left|\left\langle f-g, a_{n}\right\rangle\right|^{2} \\
= & \sum_{n=1}^{\infty} \frac{1}{2^{n}}\left(t^{2}\left|\left\langle f, a_{n}\right\rangle\right|^{2}+2 t(1-t)\left\langle f, a_{n}\right\rangle\left\langle g, a_{n}\right\rangle+(1-t)^{2}\left|\left\langle g, a_{n}\right\rangle\right|^{2}\right) \\
& +t(1-t) \sum_{n=1}^{\infty}\left(\left|\left\langle f, a_{n}\right\rangle\right|^{2}-2\left\langle f, a_{n}\right\rangle\left\langle g, a_{n}\right\rangle+\left|\left\langle g, a_{n}\right\rangle\right|^{2}\right) \\
= & t \sum_{n=1}^{\infty} \frac{1}{2^{n}}\left|\left\langle f, a_{n}\right\rangle\right|^{2}+(1-t) \sum_{n=1}^{\infty} \frac{1}{2^{n}}\left|\left\langle g, a_{n}\right\rangle\right|^{2} \\
= & t[f]^{2}+(1-t)[g]^{2}
\end{aligned}
$$

Por lo tanto, la función $f \rightarrow\|f\|^{2}+[f]^{2}$ es estrictamente convexa

Ahora sea $x \in E$ y considere

$$
\|x\|_{2}=\left(\|x\|_{1}+\sum_{n=1}^{\infty} \frac{1}{2^{n}}\left|\left\langle b_{n}, x\right\rangle\right|^{2}\right)^{1 / 2},
$$

donde $\|x\|_{1}=\sup _{\|f\|_{1} \leq 1}|\langle f, x\rangle|$

Afirmación 4: $\|.\|_{2}$ es una norma equivalente a $\|$.$\| . De manera análoga a la afirmación 1$ se prueba que $\|.\|_{2}$ es una norma en $E$. En este caso se debe notar que; debido a la densidad de $\left(b_{n}\right)$ en $E^{*}$, se obtiene

$$
\left\langle b_{n}, x\right\rangle=0 \forall n \in \mathbb{N} \Longrightarrow x=0 .
$$

Ademas

$$
\begin{aligned}
\|x\| & =\|x\|_{1}=\sup _{\|f\|_{1} \leq 1}|\langle f, x\rangle| \\
& \leq\left(\|x\|_{1}+\sum_{n=1}^{\infty} \frac{1}{2^{n}}\left|\left\langle b_{n}, x\right\rangle\right|^{2}\right)^{1 / 2}=\|x\|_{2} \\
& \leq\left(\|x\|_{1}+\sum_{n=1}^{\infty} \frac{1}{2^{n}}\left\|b_{n}\right\|^{2}\|x\|_{1}^{2}\right)^{1 / 2} \\
& \leq \sqrt{2}\|x\|_{1}=\sqrt{2}\|x\| .
\end{aligned}
$$

Afirmación 5: La norma dual de $\|\cdot\|_{2}$ es estrictamente convexa. En efecto, sea

$$
\begin{aligned}
\Phi: E & \rightarrow \mathbb{R} \\
x & \rightarrow \Phi(x)=\frac{1}{2}[x]^{2}=\frac{1}{2} \sum_{n=1}^{\infty} \frac{1}{2^{n}}\left|\left\langle b_{n}, x\right\rangle\right|^{2},
\end{aligned}
$$


puesto que [.] es inducida por un producto interno, se tiene que

$$
\Phi(y)=\Phi^{*}(y)
$$

donde $\Phi^{*}$ es la conjugada de Fenchel de $\Phi$ (ver [3]). Por lo tanto $\Phi^{*}$ es una norma. Denotemos por $\Phi^{*}()=$. [.] ${ }^{*}$ la norma conjugada de [.] en $E^{*}$, es decir

$$
[f]^{*}=\sup _{x \in E}\left\{\langle f, x\rangle-\frac{1}{2}[x]^{2}\right\}, \quad f \in E^{*} .
$$

Ahora notemos que

$$
\begin{gathered}
\left([t f+(1-t) g]^{*}\right)^{2}=\sup _{x \in E}\left\{\langle t f+(1-t) g, x\rangle-\frac{1}{2}[x]^{2}\right\} \\
\left([f-g]^{*}\right)^{2}=\sup _{y \in E}\left\{\langle f-g, y\rangle-\frac{1}{2}[y]^{2}\right\},
\end{gathered}
$$

luego,

$$
\begin{aligned}
&\left([t f+(1-t) g]^{*}\right)^{2}+t(1-t)\left([f-g]^{*}\right)^{2}=\sup _{x \in E}\left\{\langle t f+(1-t) g, x\rangle-\frac{1}{2}[x]^{2}\right\} \\
&+t(1-t) \sup _{y \in E}\left\{\langle f-g, y\rangle-\frac{1}{2}[y]^{2}\right\} \\
&=\sup _{x, y \in E}\left\{\langle t f+(1-t) g, x\rangle+t(1-t)\langle f-g, y\rangle-\frac{1}{2}[x]^{2}-\frac{1}{2} t(1-t)[y]^{2}\right\} .
\end{aligned}
$$

Haciendo el cambio de variable $x=t \xi+(1-t) \eta$ y $y=\xi-\eta$ se obtiene

$$
\langle t f+(1-t) g, t \xi+(1-t) \eta\rangle=t^{2}\langle f, \xi\rangle+t(1-t)\langle f, \eta\rangle+t(1-t)\langle g, \xi\rangle+(1-t)^{2}\langle g, \eta\rangle,
$$

$$
\begin{gathered}
t(1-t)\langle f-g, \xi-\eta\rangle=t(1-t)\langle f, \xi\rangle-t(1-t)\langle f, \eta\rangle-t(1-t)\langle g, \xi\rangle+t(1-t)\langle g, \eta\rangle \\
{[t \xi+(1-t) \eta]^{2}=\sum_{n=1}^{\infty} \frac{1}{2^{n}}\left|\left\langle b_{n}, t \xi+(1-t) \eta\right\rangle\right|^{2}} \\
=\sum_{n=1}^{\infty} \frac{1}{2^{n}}\left(t^{2}\left|\left\langle b_{n}, \xi\right\rangle\right|^{2}+2 t(1-t)\left\langle b_{n}, \xi\right\rangle\left\langle b_{n}, \eta\right\rangle+(1-t)^{2}\left|\left\langle b_{n}, \eta\right\rangle\right|^{2}\right) \\
=t^{2}[\xi]^{2}+2 t(1-t) \sum_{n=1}^{\infty} \frac{1}{2^{n}}\left\langle b_{n}, \xi\right\rangle\left\langle b_{n}, \eta\right\rangle+(1-t)^{2}[\eta]^{2} \\
{[\xi-\eta]^{2}=\sum_{n=1}^{\infty} \frac{1}{2^{n}}\left|\left\langle b_{n}, \xi-\eta\right\rangle\right|^{2}} \\
=[\xi]^{2}-2 \sum_{n=1}^{\infty} \frac{1}{2^{n}}\left\langle b_{n}, \xi\right\rangle\left\langle b_{n}, \eta\right\rangle+[\eta]^{2} .
\end{gathered}
$$

Ahora, reemplazando (2.4)-(2.7) en (2.3) se obtiene

$$
\left([t f+(1-t) g]^{*}\right)^{2}+t(1-t)\left([f-g]^{*}\right)^{2}=t[f]^{* 2}+(1-t)[g]^{* 2} .
$$

Ahora recordando que $(f+g)^{*}=f^{*} \circ g^{*}$ donde $f^{*}\left(x^{*}\right)=\sup _{x \in E}\left\{\left\langle x^{*}, x\right\rangle-f(x)\right\}$ es la conjugada de Fenchel de la función $f$ y $f \circ g=\inf _{y \in E}\{f(x-y)+g(y)\}, x \in E$ es la inf-convolución de $f$ y $g$, luego

$$
\|f\|_{2}^{* 2}=\inf _{h \in E^{*}}\left\{\|f-h\|_{1}^{2}+[h]^{* 2}\right\}=\min _{h \in E^{*}}\left\{\|f-h\|_{1}^{2}+[h]^{* 2}\right\}
$$


Ahora se demostrará que $f \rightarrow\|f\|_{2}^{* 2}$ es estrictamente convexa. En efecto, sean $f, g \in E^{*}$ fijos y $h_{1}, h_{2} \in E^{*}$ tal que

$$
\|f\|_{2}^{* 2}=\left\|f-h_{1}\right\|_{1}^{2}+\left[h_{1}\right]^{* 2}, \quad\|g\|_{2}^{* 2}=\left\|g-h_{2}\right\|_{1}^{2}+\left[h_{2}\right]^{* 2} .
$$

Luego, para todo $t n(0,1)$ se tiene

$$
\begin{aligned}
\|t f+(1-t) g\|_{2}^{* 2} & =\inf _{h \in E^{*}}\left\{\|f-h\|_{1}^{2}+[h]^{* 2}\right\} \\
& \leq\left\|t f+(1-t) g-\left(t h_{1}+(1-t) h_{2}\right)\right\|_{1}^{2}+\left[t h_{1}+(1-t) h_{2}\right]^{* 2} \\
& <t\|f\|_{2}^{* 2}+(1-t)\|g\|_{2}^{*^{2}}
\end{aligned}
$$

a menos que $f-h_{1}=g-h_{2}$ y $h_{1}=h_{2}$, es decir $f=g$, lo cual implica que $f \rightarrow\|f\|_{2}^{* 2}$ es estrictamente convexa.

3. Conclusión. Como conclusión final, se puede decir que en todo espacio de Banach separable es posible construir una norma equivalente a la original que es estrictamente convexa y cuya norma conjugada dual también es estrictamente convexa.

Referencias

[1] H. Brezis, Functional Analysis, Sobolev Spaces and Partial Differential Equations, Springer Science + Business Media, LLC 2011.

[2] G. Botelho, D. Pellegrino and E. Teixeira, "Fundamentos de Análise Funcional", EDITORASBM, Brasil, 2012.

[3] F. Álvarez, "Análisis Convexo y Dualidad”, DIM-CMM, Universidad de Chile, 2012. 